\title{
Database Access Strategy for TV White Space Cognitive Radio Networks
}

\author{
Marcello Caleffi, Angela Sara Cacciapuoti \\ Dept. of Electrical Engineering and Information Technologies \\ University of Naples Federico II, Italy \\ Email: marcello.caleffi@unina.it, angelasara.cacciapuoti@unina.it
}

\begin{abstract}
Very recently, city administrations launched TV White Space wireless networks to enable the smart city paradigm. These initiatives leverage the possibility allowed by regulations of unlicensed access to the TV White Space spectrum. All theses rulings rely on a periodic access to a database service as the primary mechanism for the unlicensed users to determine the White Space availability. Nevertheless, the specifics of such a mechanism are yet to be determined. In this paper, we address this issue by designing a database access strategy that allows unlicensed users to: $i$ ) respect the requirements imposed by the existing rulings; $i i$ ) maximize the expected communication opportunities provided by the TV White Space through the ondemand database accesses. To this aim, we prove the database access problem can be modeled as a Markov Decision Process and we provide the closed-form expressions of the transition probabilities. The analytical results are finally validated through simulations.
\end{abstract}

Index Terms-Spectrum Sharing, TV White Space, Cognitive Radio, Database, Strategy.

\section{INTRODUCTION}

Very recently, city and county administrations, such as Wilmington and New Hanover County [1], launched TV White Space wireless networks to enable the smart city paradigm, through citizens broadband wireless access, remote monitoring and controlling of facilities and traffic congestion. These initiatives leverage the possibility allowed by regulations and standards within the world of unlicensed access to the TV White Space spectrum [2], [3], [4].

All the existing rulings obviated the spectrum sensing [5], [6], [7] as the mechanism for the unlicensed users to determine the White Space availability. Instead, they require the unlicensed users to periodically access to a geolocated database service for acquiring the spectrum availability, with a fixed timeframe, referred to as database access period. In addition, the rulings allow the unlicensed user to access to the database service on-demand within the database access period, whenever it believes convenient to update the spectrum availability information.

The research leading to these results has been supported by the SHERPA collaborative project, which has received funding from the European Communitys Seventh Framework Programme (FP7/2007-2013) under grant agreement ICT-600958. The authors are solely responsible for its content. It does not represent the opinion of the European Community, that is not responsible for any use that might be made of the information contained therein.

978-1-4799-4657-0/14/\$31.00 (C) 2014 IEEE
Clearly, the database access strategy is a key factor for the performance of any Cognitive Radio (CR) network operating within the TV White Space, although the particulars of the database access strategy are yet to be determined. Specifically, any ruling specifies the database access period (usually 24 or 48 hours), but the specifics of the on-demand accesses within a database period are never detailed.

Hence, in this paper, we address such an issue by designing the optimal database access strategy, i.e., the database access strategy that allows the CR users to: $i$ ) respect the requirements imposed by the existing rulings in term of periodic mandatory access to the database service; $i i)$ maximize the expected TV White Space communication opportunities through the on-demand accesses to the database service.

To this aim, at first, we develop an analytical framework through which the choice of the database access strategy is modeled as a decision process, by deriving the CR user states and the corresponding set of actions available to the $\mathrm{CR}$ user. Then, we prove that the problem of choosing the optimal database access strategy can be modeled as a Markov Decision Process, and we provide closed-form expressions of the transition probabilities. Finally, we derive the optimal database access strategy. The theoretical results are validated through a case-study.

To the best of our knowledge, this is the first work addressing the problem of selecting the database access strategy for TV White Space according to the existing rulings.

The rest of the paper is organized as follows. In Sec. II, we describe the network model along with some preliminaries. In Sec. III we derive the analytical framework for the optimal database access strategy, and we validate it through a case study in Sec. IV. In Sec. V we conclude the paper, and, finally, some proofs are gathered in the appendix.

\section{Network Model ANd Preliminaries}

In this section, we first describe the system model in Sec. II-A. Then, in Sec. II-B, we collect several definitions that will be used through the paper.

\section{A. System Model}

We consider a CR network operating in the TV white space spectrum.

The spectrum is organized in $M$ distinct bands (channels), denoted with the set $\Omega=\{1,2, \ldots, M\}$. The PU traffic on 
channel $i \in \Omega$ is modeled as a Bernoulli processes with parameter $p_{i}$ denoting the PU inactivity probability. Specifically, the channel status process ${ }^{1} S_{i}(n)=1$ denotes the availability of the $i$-th channel to the CR network in $n$-th time slot, and it results $p_{i}=P\left\{S_{i}(n)=1\right\}$ for any time slot $n$. Furthermore, $N_{i}(n)=n_{i}$ denotes the availability of the $i$-th channel to the CR networks for $n_{i}$ consecutive time slots starting at the $n$-th time slot, and it results $P\left\{N_{i}(n)=n_{i}\right\}=p_{i}^{n_{i}} \forall n$.

According to current active regulations [10], [9] and standard [8], a CR user operates according to the following rules:

Rule 1. Each CR user must periodically ${ }^{2}$ access to a database service to obtain the spectrum availability information.

Rule 2. Within a database access period, each CR user can access on-demand to a database service to update the spectrum availability information.

Rule 3. The spectrum availability information consists of a list of channels within which the CR user is authorized to operate and, for each channel, the duration of such an authorization.

Remark. The spectrum availability information consists of the channel statuses $\left\{S_{i}(n)\right\}_{i \in \Omega}$ and the channel availabilities $\left\{N_{i}(n)\right\}_{i \in \Omega}{ }^{3}$.

\section{B. Assumptions and Definitions}

In the following, we give some assumptions and definitions adopted through the paper.

Assumption 1. (Cognitive User Time) The CR user time is organized into $N$ slots of duration $T$, with $K T$ denoting the database access period, i.e., the maximum time interval between two mandatory database accesses (Rule 1).

Remark. Physically, the time horizon $N$ represents the time interval during which the CR user plans to opportunistically use the TV White Space spectrum.

Definition 1. (Action Set) The action set $A$ is the set of actions available at the $\mathrm{CR}$ user:

$$
A=\left\{a_{-1}, a_{1}, \ldots, a_{M}\right\}
$$

where:

- Action $a_{-1}$ denotes the event "the CR user updates the spectrum availability information in the current time slot by accessing the database" (Rules 1 and 2).

- Action $a_{j}, j \in \Omega$, denotes the event "the CR user accesses to the $j$-th channel for packet transmission in the current time slot".

${ }^{1}$ We assume the channel statuses $S_{i}(n)$ and $S_{j}(n)$ independent $\forall i \neq j$. Such an assumption is not restrictive, since when the channel statuses are correlate, e.g., due to adjacent channel use restriction [8], [9], $S_{i}(n)$ represents either the status of the best channel belonging to a set of correlated channels (if the CR user is equipped with a single radio interface) or the status of a set of correlated channels (if the CR user is equipped with multiple radio interfaces).

${ }^{2}$ IEEE 802.22 and OfComm requires at least one database access every 24 hours (see 10.6.2 in [8] and 3.7 in [9]), whereas FCC requires at least one database access every 48 hours (see $15.711 . b .3$ in [10]).

${ }^{3}$ See 15.711.b.3 in [10], 3.23 in [9], and 10.7.1.6 in [8]
Definition 2. (Cognitive User State) At the $n$-th time slot, the $C R$ user state is defined as the pair:

$$
\left(\tilde{n}_{n}, \mathbf{d}_{n}\right)
$$

where $\tilde{n}_{n}$ denotes the time slot of the previous database access at time slot $n$, and $\mathbf{d}_{n}=\left(d_{n}^{1}, \ldots, d_{n}^{M}\right)$ denotes the $M$-ple defined as follows (Rule 3):

$$
d_{n}^{i}= \begin{cases}n_{i} & \text { if } S_{i}\left(\tilde{n}_{n}\right)=1 \wedge N_{i}\left(\tilde{n}_{n}\right)=n_{i}, \tilde{n}_{n}+n_{i} \geq n \\ 0 & \text { otherwise }\end{cases}
$$

with $S_{i}\left(\tilde{n}_{n}\right)$ denoting the realization of the $i$-th channel status process acquired through a database access at time slot $\tilde{n}_{n}$, and $N_{i}\left(\tilde{n}_{n}\right)=n_{i}$ denoting the realization of the $i$-th channel availability process acquired through a database access at time slot $\tilde{n}_{n}$. In the following, we denote with $\Sigma$ the set of CR user states.

Remark. At the $n$-th time slot, $d_{n}^{i} \neq 0$ if and only if:

a) at time slot $\tilde{n}_{n}$ the database revealed channel $i$ as available for the subsequent $n_{i}$ time slots;

b) the channel $i$ is still available at time slot $n$, i.e., $\tilde{n}_{n}+$ $n_{i} \geq n$.

Definition 3. (Allowed Action Set) The allowed action set $A_{\left(\tilde{n}_{n}, \mathbf{d}_{n}\right)}$ is the set of actions available at the CR user when the CR user state is $\left(\tilde{n}_{n}, \mathbf{d}_{n}\right)$ at time slot $n$ :

$$
A_{\left(\tilde{n}_{n}, \mathbf{d}_{n}\right)}= \begin{cases}\left\{a_{-1}\right\} & \text { if } \tilde{n}_{n}+K<n \\ \left\{a_{-1}\right\} \cup\left\{a_{j}: d_{j} \neq 0\right\} & \text { if } \tilde{n}_{n}+K \geq n\end{cases}
$$

Remark. According to Definition 1, $a_{j} \in A_{\left(\tilde{n}_{n}, \mathbf{d}_{n}\right)}, a_{j} \neq a_{-1}$ whenever:

a) the $j$-th channel is available at time slot $n$, i.e., $d_{j} \neq 0$;

b) the mandatory database access requirement is satisfied, i.e., $\tilde{n}_{n}+K \geq n$.

Remark. Since $a_{-1} \in A_{\left(\tilde{n}_{n}, \mathbf{d}_{n}\right)} \forall \mathbf{d}_{n}$, the CR user operates in agreement with Rule 2, i.e., the CR user can access to the database service on-demand. Furthermore, since we assume $N_{i}\left(\tilde{n}_{n}\right)=n_{i} \leq K$ for any $i \in \Omega$ and for any $\tilde{n}_{n}$, the CR user operates in agreement with Rule 1, i.e., the CR user accesses to the database service at most every $K$ time slots.

Assumption 2. (Reward and Cost) We model the communication quality of the $i$-th channel ${ }^{4}$ with the dimensionless quantity $r_{i}$, referred to as channel reward. Furthermore, we model the overhead induced by a database access with the dimensionless quantity $c$, referred to as database access cost.

Remark. By abstracting the communication opportunities and overheads from the particulars through the general notions of reward and cost, the adopted model achieves the following two key features: a) it restricts our attention on the effects of the database access strategy; b) it allows us to measure the performance of a database access strategy, and thus to quantitatively compare different strategies.

\footnotetext{
${ }^{4}$ If the CR user is equipped with multiple radio interfaces, then the reward $r_{i}$ denotes the cumulative reward provided by a set of channels.
} 
Definition 4. (Reward) By choosing action $a \in A_{\left(\tilde{n}_{n}, \mathbf{d}_{n}\right)}$ when the CR user state is $\left(\tilde{n}_{n}, \mathbf{d}_{n}\right)$ at time slot $n$, the CR user reward $r_{a}\left(\tilde{n}_{n}, \mathbf{d}_{n}\right)$ is:

$$
r_{a}\left(\tilde{n}_{n}, \mathbf{d}_{n}\right)= \begin{cases}-c & \text { if } a=a_{-1} \\ r_{i} & \text { if } a=a_{i}, i \in \Omega\end{cases}
$$

Assumption 3. (Ordered Channel Set) We assume the channel set $\Omega$ ordered according to the channel rewards:

$$
r_{i} \geq r_{i-1} \quad \forall i=2, \ldots, M
$$

\section{Optimal Database Access Strategy}

Here, we formulate the database access problem by proving that the problem of choosing the database access strategy can be modeled as a Markov Decision Process, where the reward models the discovered communication opportunities and the cost models the overhead associated with a database access (Theorem 1). Theorem 1, requires the preliminary result stated in Lemma 1.

Lemma 1. (Transition Probability) Given that the CR user chooses action $a \in A_{\left(\tilde{n}_{n}, \mathbf{d}_{n}\right)}$, when the $C R$ user state is $\left(\tilde{n}_{n}, \mathbf{d}_{n}\right)$ at time slot $n$, the probability of the CR user state being $\left(\tilde{n}_{n+1}, \mathbf{d}_{n+1}\right)$ at time slot $n+1$ is conditionally independent of all the previous states and actions, and it results (7) shown in the next page, where $p_{a}\left(\tilde{n}_{n+1}, \mathbf{d}_{n+1} \mid \tilde{n}_{n}, \mathbf{d}_{n}\right)$ denotes the transition probability when action a is chosen.

Proof: See Appendix A.

Theorem 1. The database access problem is a Markov Decision Process.

Proof: The proof follows from Lemma 1 by accounting for the Markov property of the transition probabilities [11].

Definition 5. (Strategy) A strategy $\pi$ is a function that maps the set of CR user states over the set of allowed actions:

$$
\forall\left(\tilde{n}_{n}, \mathbf{d}_{n}\right) \in \Sigma: \pi\left(\tilde{n}_{n}, \mathbf{d}_{n}\right) \in A_{\left(\tilde{n}_{n}, \mathbf{d}_{n}\right)}
$$

In the following, we denote with $\Pi$ the set of strategies.

Corollary 1. (Expected Total Reward) Given the time horizon $N$, the expected total reward $v_{\pi}(0, \mathbf{0})$ obtained by the $C R$ user starting from the state $(0,0)$ at time slot 1 and following the strategy $\pi$ is equal to:

$v_{\pi}(0, \mathbf{0})=r_{a_{-1}}(0, \mathbf{0})+\sum_{\left(\tilde{n}_{2}, \mathbf{d}_{2}\right) \in \Sigma} p_{a_{-1}}\left(\tilde{n}_{2}, \mathbf{d}_{2} \mid 0, \mathbf{0}\right) v_{\pi}\left(\tilde{n}_{2}, \mathbf{d}_{2}\right)$

where $\mathbf{0}=(0, \ldots, 0)$ denotes the null $M$-ple, and $v_{\pi}\left(\tilde{n}_{n}, \mathbf{d}_{n}\right)$ denotes the expected remaining reward at time slot $n$ defined in (10) shown in the next page, i.e., it denotes the reward obtained by the CR user starting from the state $\left(\tilde{n}_{n}, \mathbf{d}_{n}\right)$ at time slot $n$ and following the strategy $\pi$. In (10) $p_{\pi\left(\tilde{n}_{n}, \mathbf{d}_{n}\right)}\left(\tilde{n}_{n+1}, \mathbf{d}_{n+1} \mid \tilde{n}_{n}, \mathbf{d}_{n}\right)$ denotes the transition probability when action $\pi\left(\tilde{n}_{n}, \mathbf{d}_{n}\right)$ is chosen according to strategy $\pi$.
Proof: According to Theorem 1, the optimal database access problem is a Markov Decision Process. Hence, the proof follows from Theorem 4.2 in [11].

Remark. The expected total reward $v_{\pi}(0,0)$ has been defined as a recursive function. More in detail, $v_{\pi}\left(\tilde{n}_{n}, \mathbf{d}_{n}\right)$ at the recursive step $n$ is function of: i) the CR user state $\left(\tilde{n}_{n}, \mathbf{d}_{n}\right)$; ii) the strategy $\pi$ through the action $\pi\left(\tilde{n}_{n}, \mathbf{d}_{n}\right)$; iii) the reward $v_{\pi}\left(\tilde{n}_{n+1}, \mathbf{d}_{n+1}\right)$ obtained at the next time slot through the transition probability $p_{\pi\left(\tilde{n}_{n}, \mathbf{d}_{n}\right)}\left(\tilde{n}_{n+1}, \mathbf{d}_{n+1} \mid \tilde{n}_{n}, \mathbf{d}_{n}\right)$. At time instant $n=1$ the only allowed action is $a_{-1}$ since no spectrum opportunities have been previously discovered, i.e., the CR user state is $(0, \mathbf{0})$.

Definition 6. (Optimal Database Access Problem) Given the channel set $\Omega=\{1,2, \ldots, M\}$, the statistics on the channel statuses $\left\{S_{i}(n)\right\}_{i \in \Omega}$ and the channel availabilities $\left\{N_{i}(n)\right\}_{i \in \Omega}$, the channel rewards $\left\{r_{i}\right\}_{i \in \Omega}$ and the database access $\operatorname{cost} c$, the time horizon $N$ and the mandatory database access parameter $K$, the goal is to choose the strategy $\pi^{*} \in \Pi$ that maximizes the expected total reward:

$$
v_{\pi^{*}}(0, \mathbf{0})=\sup _{\pi \in \Pi}\left\{v_{\pi}(0, \mathbf{0})\right\}
$$

and we refer to $\pi^{*}$ as the optimal strategy.

Corollary 2. Given the channel set $\Omega=\{1,2, \ldots, M\}$, the statistics on the channel statuses $\left\{S_{i}(n)\right\}_{i \in \Omega}$ and the channel availabilities $\left\{N_{i}(n)\right\}_{i \in \Omega}$, the channel rewards $\left\{r_{i}\right\}_{i \in \Omega}$ and the database access cost $c$, the time horizon $N$ and the mandatory database access parameter $K$, the optimal database access strategy is the solution of the following problem:

$$
v_{\pi^{*}}(0, \mathbf{0})=\max _{\pi \in \Pi}\left\{v_{\pi}(0, \mathbf{0})\right\}
$$

Proof: According to Theorem 1, the optimal database access problem is a Markov Decision Process. Hence, since the sets $\Sigma$ and $A$ are finite, it always exists a deterministic strategy achieving the supremum in (11) (Lemma 4.3.1 in [11]).

Remark. Solving (12) with brute-force search is computational unfeasible. Hence, in [12] we derive a computationalefficient algorithm by proving that the optimal database access strategy exhibits a threshold structure.

\section{Case Study}

Here, we validate the proposed optimal database access algorithm for TV White Space spectrum sharing. More specifically, we simulate a CR network operating in the TV White Space according to Rules 1-3 and we evaluate its performance in terms of expected total reward (9) as a function of the adopted strategy. The adopted simulation set is as follows: $M=3, K=3, N=30$, and $c=0.001$.

In Fig. 1 we report the expected total reward as a function of the discrete time for $p_{i}=0.1 ; \forall i \in \Omega$. More in detail, we consider the following rewards: i) the Optimal Strategy Reward, i.e., the reward achieved by the strategy defined in (12); ii) the Average Reward, i.e., the reward achievable by 


$$
\begin{aligned}
& p_{a_{-1}}\left(\tilde{n}_{n+1}, \mathbf{d}_{n+1} \mid \tilde{n}_{n}, \mathbf{d}_{n}\right)= \begin{cases}\prod_{d_{n+1}^{i} \neq 0} p_{i}^{k_{i}} \prod_{d_{n+1}^{j}=0} \bar{p}_{j} & \text { if } \begin{cases}\tilde{n}_{n+1}=n+1 \\
k_{i}=\left\{\begin{array}{l}
d_{n+1}^{i} \\
n+1+d_{n+1}^{i}-\tilde{n}_{n}-d_{n}^{i}
\end{array}\right. & \text { if } \tilde{n}_{n}+d_{n}^{i}<n+1 \\
\tilde{n}_{n}+d_{n}^{j}<n+1 & \text { otherwise }\end{cases} \end{cases} \\
& p_{a \neq a_{-1}}\left(\tilde{n}_{n+1}, \mathbf{d}_{n+1} \mid \tilde{n}_{n}, \mathbf{d}_{n}\right)=\left\{\begin{array}{l}
\text { if }\left\{\begin{array}{l}
\tilde{n}_{n+1}=\tilde{n}_{n} \\
d_{n+1}^{i}
\end{array}= \begin{cases}d_{n}^{i} & \text { if } \tilde{n}_{n}+d_{n}^{i} \geq n+1 \\
0 & \text { otherwise }\end{cases} \right. \\
0 \quad \text { otherwise }
\end{array}\right. \\
& v_{\pi}\left(\tilde{n}_{n}, \mathbf{d}_{n}\right)= \begin{cases}r_{\pi\left(\tilde{n}_{n}, \mathbf{d}_{n}\right)}\left(\tilde{n}_{n}, \mathbf{d}_{n}\right)+\sum_{\left(\tilde{n}_{n+1}, \mathbf{d}_{n+1}\right) \in \Sigma} p_{\pi\left(\tilde{n}_{n}, \mathbf{d}_{n}\right)}\left(\tilde{n}_{n+1}, \mathbf{d}_{n+1} \mid \tilde{n}_{n}, \mathbf{d}_{n}\right) v_{\pi}\left(\tilde{n}_{n+1}, \mathbf{d}_{n+1}\right) & \text { if } 1<n<N \\
r_{\pi\left(\tilde{n}_{N}, \mathbf{d}_{N}\right)}\left(\tilde{n}_{N}, \mathbf{d}_{N}\right) & \text { if } n=N\end{cases}
\end{aligned}
$$

averaging over all the considered admissible strategies; ii) the Non-Optimal Strategy Reward, i.e., the reward achievable by an arbitrary admissible strategy different from the optimal strategy. We limit the number of considered strategies to 65 for the sake of figure legibility.

First, we note that, in any time slot different from 1 the figure shows the expected remaining reward at that time slot. Furthermore, we note that the expected remaining reward decreases as the time slot increases, and this is reasonable and in agreement with expression (10). Finally, we note that the choice of the database access strategy deeply affects the overall performance, since the optimal database strategy achieves an expected total reward significantly higher then the average reward.

To better understand the effect of the channel availabilities on the achievable rewards, in Fig. 2 we present the expected total reward as a function of the discrete time for $p_{i}=0.01 ; \forall i \in \Omega$. We observe that the lower is the probability $p_{i}$ of channel $i$ being available, the lower is the expected total reward, but the difference between the optimal strategy reward and the average reward remains significative.

\section{CONCLUSIONS}

Very recently, several regulations and standards have approved or are underway to approve the dynamic access of unlicensed users to the TV White Space spectrum. All the existing rulings rely on a periodic access to a database service as the primary mechanism for the unlicensed users to determine the White Space availability. In this paper, we addressed the problem of accessing to the database service by designing an optimal database access strategy, i.e., by designing a strategy allowing the unlicensed users to jointly: i) respect the requirements imposed by the existing rulings in term of periodic mandatory access to the database service; ii) maximize the expected TV White Space communication opportunities through on-demand accesses to the database service. Specifically, we developed an analytical framework through which the choice of the database access strategy is modeled as a decision process. Moreover, we proved that the problem of choosing the optimal database access strategy can be modeled as a Markov Decision Process, and we provided closed-form expressions of the transition probabilities. The numerical validation of the proposed strategy through a case study confirmed the optimality property.

\section{APPENDIX}

\section{A. Proof of Lemma 1}

Proof:

Case 1: $a \neq a_{0}$. By choosing action $a \neq a_{0}$ at time slot $n$, the $\mathrm{CR}$ user does not acquire any updated spectrum availability information. Hence, the next CR user state $\left(\tilde{n}_{n+1}, \mathbf{d}_{n+1}\right)$ is univocally determined by $\left(\tilde{n}_{n}, \mathbf{d}_{n}\right)$. Specifically, by accounting for Definition 2, it results:

$$
\begin{aligned}
& \tilde{n}_{n+1}=\tilde{n}_{n} \\
& d_{n+1}^{i}= \begin{cases}d_{n}^{i} & \text { if } \tilde{n}_{n}+d_{n}^{i} \geq n+1 \\
0 & \text { otherwise }\end{cases}
\end{aligned}
$$

and the thesis follows.

Case 2: $a=a_{0}$. By choosing action $a_{0}$ at time slot $\mathrm{n}$, the $\mathrm{CR}$ user does update the spectrum availability information. Hence, it results $\tilde{n}_{n+1}=n+1$. Furthermore, with reference to the $i$-th channel, we have $d_{n+1}^{i} \neq 0$ if and only if $S_{i}(n+1)=1$ and $N_{i}(n+1)=d_{n+1}^{i}$. We have two cases [13], [14]: i) if $\tilde{n}_{n}+d_{n}^{i} \geq n+1$, i.e., if during the previous database access at time slot $\tilde{n}_{n}$ channel $i$ was reported as available up to time slot $n+1$, then $P\left\{N_{i}(n+1)=d_{n+1}^{i} \mid N_{i}\left(\tilde{n}_{n}\right)=d_{n}^{i}\right\}=$ $p_{i}^{k_{i}}, k_{i}=n+1+d_{n+1}^{i}-\tilde{n}_{n}-d_{n}^{i}$; ii) otherwise, $P\left\{N_{i}(n+1)=\right.$ $\left.d_{n+1}^{i} \mid N_{i}\left(\tilde{n}_{n}\right)=d_{n}^{i}\right\}=P\left\{N_{i}(n+1)=d_{n+1}^{i}\right\}=p_{i}^{d_{n+1}^{i}}$. Finally, we have $d_{n+1}^{i}=0$ if and only if $S_{i}(n+1)=0$ and $\tilde{n}_{n}+d_{n}^{i}<n+1$, i.e., if during the previous database access 


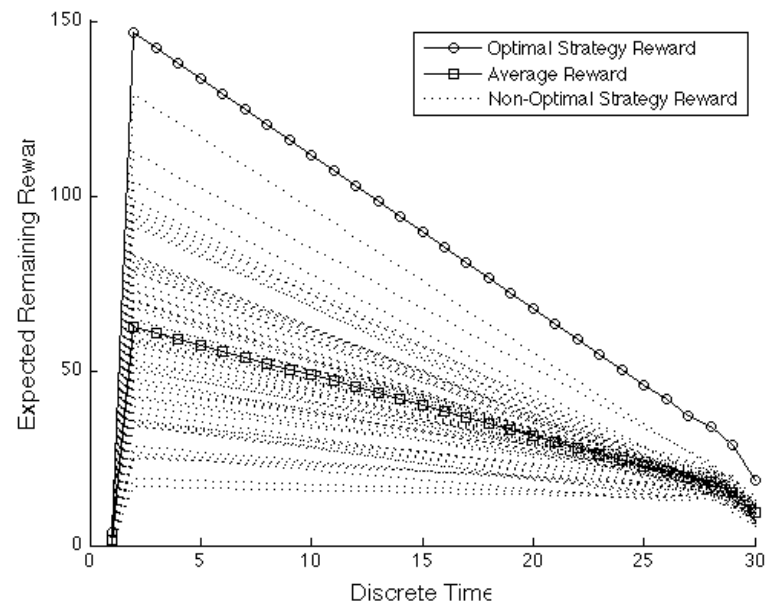

Fig. 1. Expected remaining reward vs discrete time for $p_{i}=0.1 \forall i \in \Omega$.

at time slot $\tilde{n}_{n}$ channel $i$ was not reported as available up to time slot $n+1$.

\section{REFERENCES}

[1] K. Shin, H. Kim, A. Min, and A. Kumar, "Cognitive radios for dynamic spectrum access: from concept to reality," Wireless Communications, IEEE, vol. 17, no. 6, pp. 64-74, December 2010.

[2] M. Nekovee, T. Irnich, and J. Karlsson, "Worldwide trends in regulation of secondary access to white spaces using cognitive radio," IEEE Wireless Communications, vol. 19, no. 4, pp. 32-40, August 2012.

[3] A. Flores, R. Guerra, E. Knightly, P. Ecclesine, and S. Pandey, "Ieee 802.11af: a standard for tv white space spectrum sharing," IEEE Communications Magazine, vol. 51, no. 10, pp. 92-100, October 2013.

[4] C.-S. Sum, G. Villardi, M. Rahman, T. Baykas, H. N. Tran, Z. Lan, C. Sun, Y. Alemseged, J. Wang, C. Song, C. woo Pyo, S. Filin, and H. Harada, "Cognitive communication in tv white spaces: An overview of regulations, standards, and technology," IEEE Communications Magazine, vol. 51, no. 7, pp. 138-145, July 2013.

[5] A. S. Cacciapuoti, M. Caleffi, D. Izzo, and L. Paura, "Cooperative spectrum sensing techniques with temporal dispersive reporting channels," IEEE Transactions on Wireless Communications, vol. 10, no. 10, pp. 3392-3402, October 2011.

[6] I. F. Akyildiz, B. F. Lo, and R. Balakrishnan, "Cooperative spectrum sensing in cognitive radio networks: A survey," Elsevier Physical Communication, vol. 4, no. 1, pp. $40-62,2011$.

[7] A. S. Cacciapuoti, M. Caleffi, L. Paura, and R. Savoia, "Decision maker approaches for cooperative spectrum sensing: Participate or not participate in sensing?" IEEE Transactions on Wireless Communications, vol. 12, no. 5, pp. 2445-2457, May 2013.

[8] 802.22 Working Group on Wireless Regional Area Networks, "Part 22: Cognitive wireless ran medium access control (mac) and physical layer (phy) specifications: Policies and procedures for operation in the tv bands," IEEE, Active Standard, 2011.

[9] OfCom, "Regulatory requirements for white space devices in the uhf tv band," OfCom, Active Regulation, July 2012.

[10] FCC, "Et docket 10-174: Second memorandum opinion and order in the matter of unlicensed operation in the tv broadcast bands," FCC, Active Regulation, September 2012.

[11] M. L. Puterman, Markov Decision Processes: Discrete Stochastic Dynamic Programming, ser. Wiley series in probability and mathematical statistics. John Wiley \& Sons, Inc., 1994.

[12] M. Caleffi and A. S. Cacciapuoti, "Optimal database access for tv white space," under review.

[13] A. S. Cacciapuoti, M. Caleffi, and L. Paura, "A theoretical model for opportunistic routing in ad hoc networks," in International Conference on Ultra Modern Telecommunications Workshops, Oct 2009, pp. 1-7.

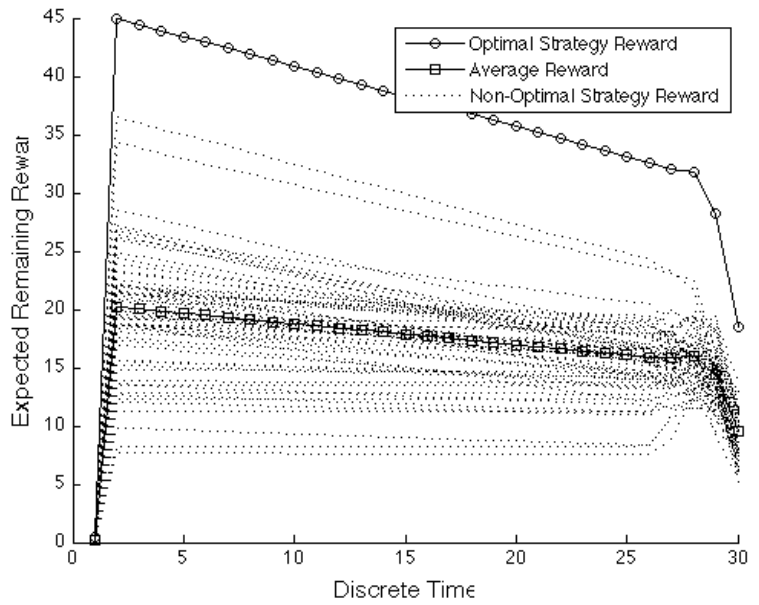

Fig. 2. Expected remaining reward vs discrete time for $p_{i}=0.01 \forall i \in \Omega$.

[14] _ "Optimal constrained candidate selection for opportunistic routing," in IEEE Global Telecommunications Conference (GLOBECOM), Dec 2010, pp. 1-5. 